\title{
Automated anesthesia artifact analysis: can machines be trained to take out the garbage?
}

\author{
Allan F. Simpao ${ }^{1,2}$ (D) Olivia Nelson ${ }^{1,2}$ (D) Luis M. Ahumada ${ }^{3,4}$
}

Received: 3 September 2020 / Accepted: 8 September 2020 / Published online: 12 September 2020

C Springer Nature B.V. 2020

The phrase "garbage in, garbage out" warns of the possibility of poor data quality input producing unreliable or nonsense output. In health care, data artifacts can lead to faulty conclusions influencing patient care guidelines, quality improvement initiatives, and research [1]. Thus, processes for both identifying artifacts and ensuring data quality should be established to reduce the potential for adverse consequences [2].

Numerous studies have both reported artifacts in perioperative vital sign data in anesthesia information management systems (AIMS) and electronic health record systems (EHRs) and described filtering methods to reduce the impact of data artifacts [3-5]. The accurate detection and identification of artifacts when analyzing perioperative data retrospectively can be challenging, as vital sign values outside of the normal range may represent the actual patient's state [6]. Meanwhile, prospective studies of perioperative data artifacts can be labor intensive and require research support that may not be widely available [6, 7].

Researchers have explored the notion of automated detection of physiological data artifacts since the proliferation of microcomputers in the 1980s [8-10]. In the early 2000s, computers were used to perform real-time artifact annotation in AIMS records, yet the use of computers for that

Allan F. Simpao

simpaoa@email.chop.edu

1 Department of Anesthesiology and Critical Care Medicine, Children's Hospital of Philadelphia, 3401 Civic Center Blvd, Philadelphia, PA 19104-4399, USA

2 Department of Anesthesiology and Critical Care, University of Pennsylvania Perelman School of Medicine, Philadelphia, PA 19104, USA

3 Department of Health Informatics, Predictive Analytics Core, Johns Hopkins All Children's Hospital, 501 6th Ave S, St. Petersburg, FL 33701, USA

4 Department of Anesthesiology and Critical Care Medicine, Johns Hopkins School of Medicine, 733 N Broadway, Baltimore, MD 21205, USA purpose remained limited to the research setting [11]. The widespread adoption of AIMS and EHRs in the 2010s gave rise to many researchers and clinicians using perioperative "big data" for quality improvement and research, with the recognition that data artifacts and poor data quality may adversely impact efforts $[1,12,13]$. Advances in computing technology over the past several years have led to artificial intelligence and machine learning being increasingly applied to large perioperative datasets for descriptive and predictive purposes [14-16]. However, few studies have applied these technologies to automated perioperative data artifact detection.

In this issue, Pasma et al. describe a study in which two research assistants performed manual identification of AIMS data artifacts, and the research team assessed the performance of three machine learning algorithms to detect artifacts [17]. This study builds upon other efforts by the authors, some of whom have been conducting research on anesthesia artifacts for nearly a decade [6, 7, 18]. Pasma et al. clearly put forth a considerable amount of time and effort in this study to perform both live and retrospective annotation of AIMS data. This attention to detail when analyzing perioperative data validity and quality is a hallmark of their previous work, and the authors emphasize continually the importance of finding ways to remove artifacts to ensure that erroneous data do not affect research findings [18].

Pasma and colleagues used techniques that other researchers might find useful when studying artifacts in their own data [17]. One challenge in studying AIMS data is aligning time stamps of perioperative events with observers' annotations, because research assistants typically do not record their observations in the AIMS. Pasma et al. used a flush event to mark the beginning of an observation period of invasive blood pressure measurements, as their annotation software was not able to register the true observation time. They focused on the maintenance phase of anesthesia to minimize the impact of the chaos of induction and emergence on the artifact recording and analysis. Pasma et al. 
studied both retrospective and live annotation of artifacts to quantify the differences between the two approaches, and they included a thoughtful discussion of the implications of their study that questioned what should and should not be considered an artifact.

Pasma and colleagues' study adds meaningfully to the growing body of literature on the application of learning algorithms to detect vital sign data artifacts. Chen et al. explored the utility of machine learning algorithms to classify alerts as real or artifacts in online noninvasive vital sign data streams and found the machine learning models discerned clinically relevant peripheral oximetry, blood pressure, and respiratory rate alerts from artifacts with an area under the curve $>0.87$ [19]. Du et al. compared the performance of a machine learning algorithm to identify artifacts in arterial line blood pressures with manual review of the dataset; they reported performance of $87 \%$ sensitivity and $99.4 \%$ specificity [20]. Hravnak et al. applied machine learning algorithms to expert-labeled vital sign data streams to classify vital sign alerts as real or artifact with reasonably accurate results [21].

The mediocre performance of the machine learning algorithms in the Pasma et al. study is an interesting finding, and the authors' approach has both pros and cons and provides meaningful insight to others who might want to apply similar tools to their own AIMS data [17]. On a positive note, Pasma et al. provide an attractive solution for using machine learning applied to physiologic data to identify and remove data artifacts accurately. The authors' effort to generalize their algorithms and thereby make them applicable and effective across a wide range of inputs was interesting and notable. Nevertheless, their approach might have benefited from adding more features as the authors recognized them (e.g., patient characteristics, procedure type, medications, and other events at the time of data artifacts). These additions might have counterbalanced the greater number of features to which the research assistants had access during the annotation process. The machine learning algorithms were provided with less information, yet their performance was matched against humans creating annotations using richer data.

It seems almost unfair to the proposed machine learning solution that the research assistants conducted their analyses during annotation using a visual approach, by looking at the event data and then assigning data validity. Thus, why not level the playing field? An alternative solution to help with model performance might consist of implementing an advanced machine learning technique such as convolutional neural network $(\mathrm{CNN})$, which is a popular approach for image recognition and classification [22]. Image representation of blood pressure data can incorporate different feature types which are not present in a one-dimensional signal. The high performance of CNNs on the classification of time-series data transformed into recurrence plots has been demonstrated [23]. For these reasons, blood pressure labeled into artifact and non-artifact groups could be converted into two-dimensional texture images using recurrence plots, and these images could then be processed through a CNN for the appropriate classification.

When identifying and removing data artifacts, it is important to keep in mind that any removal of data may eliminate potentially useful data, and data smoothing may be another possible option based on the preceding and following values surrounding an artifact value. Digital signal processing techniques can be used to process and identify artifactual or corrupted waveforms instead of removing data artifacts. For example, Thakkar et al. used a digital filter that took advantage of the blood pressure signal waveform periodic nature to identify the effect of motion artifact from blood pressure measurements, and Bose et al. used a modified version of morphological digital filtering to annotate $\mathrm{R}$ waves in electrocardiograms [24, 25]. Additionally, developing tools to extract the features of arterial blood pressure waveforms to help identify artifacts might also be of interest [26].

The authors included a comprehensive and fair discussion of their study's limitations. The exclusion of pulse oximetry was perhaps one missed opportunity to find artifacts retrospectively or with machine learning. For example, pulse oximetry data might have been used to validate the electrocardiogram heart rate data and corroborate arterial line motion artifacts. While it is understandable to exclude anesthesia induction and emergence, those two time periods are arguably the most critical clinically, and they likely have the highest occurrence of relevant vital sign deviations. It would be interesting to have a research assistant prospectively observe during those critical time periods and put machine learning to the test.

Pasma and colleagues have done an admirable job of not overstating their findings and instead have shared a stepping stone upon which similar studies can and should be built. While their study shows a machine cannot currently replace a human in the crucial, labor-intensive step of perioperative data validation, their work should inspire others to take a close look at their own data and explore how applying machine learning algorithms might be used to improve data quality. In the future, efforts in applying machine learning to perioperative data should be aimed at not only providing predictive analysis, but also detecting and addressing data artifacts so that predictions are built on high quality data [27]. Pasma and colleagues have provided an informative and encouraging step in a worthwhile, collective journey.

Authors' Contributions A.F.S., O.N., and L.M.A. contributed substantially to the conception and design of this commentary, drafted 
the article and revised it critically for important intellectual content, approved of the final version to be published, and agree to be accountable for all aspects of the work thereby ensuring that questions related to the accuracy or integrity of any part of the work are appropriately investigated and resolved.

Funding This work was supported only by departmental resources.

\section{Compliance with ethical standards}

Conflict of interests Allan F. Simpao, MD, MBI is on the Associate Editorial Board of Anesthesiology, the Journal of the American Society of Anesthesiologists. Olivia Nelson, MD has no conflicts of interest to declare. Luis M. Ahumada, PhD, MSCS has no conflicts of interest to declare.

Ethical approval This commentary was exempt from review by the Institutional Review Boards at the Children's Hospital of Philadelphia and Johns Hopkins All Children's Hospital.

\section{References}

1. Simpao AF, Ahumada LM. Rehman MA Big data and visual analytics in anaesthesia and health care. Br J Anaesth. 2015;115:350-6. https://doi.org/10.1093/bja/aeu552.

2. Kilkenny MF, Robinson KM. Data quality: garbage in-garbage out. Health Inf Manag. 2018;47:103-5. https://doi.org/10.1177/18333 58318774357.

3. Takla G, Petre JH, Doyle DJ, Horibe M, Gopakumaran B. The problem of artifacts in patient monitor data during surgery: a clinical and methodological review. Anesth Analg. 2006;103:1196-204. https:// doi.org/10.1213/01.ane.0000247964.47706.5d.

4. Eden A, Grach M, Goldik Z, et al. The implementation of an anesthesia information management system. Eur J Anaesthesiol. 2006;23:882-9. https://doi.org/10.1017/S0265021506000834.

5. Hravnak M, Chen L, Bose E, et al. Artifact patterns in continuous noninvasive monitoring of patients. Intensive Care Med. 2013;39:S405.

6. Hoorweg AJ, Pasma W, van Wolfswinkel L, de Graaff JC. Incidence of artifacts and deviating values in research data obtained from an anesthesia information management system in children. Anesthesiology. 2018;128:293-304. https://doi.org/10.1097/ALN.0000000000 001895.

7. Kool NP, van Waes JA, Bijker JB, et al. Artifacts in research data obtained from an anesthesia information and management system. Can J Anaesth. 2012;59:833-41. https://doi.org/10.1007/s1263 0-012-9754-0.

8. East KA, East TD, Mathews VJ, Waterfall BT. Computerized artifact detection for ventilatory inductance plethysmographic apnea monitors. J Clin Monit. 1989;5:170-6. https://doi.org/10.1007/BF016 27449.

9. Cluitmans PJ, Jansen JW, Beneken JE. Artifact detection and removal during auditory evoked potential monitoring. J Clin Monit. 1993;9:112-20. https://doi.org/10.1007/BF01616925.

10. Rennels GD, Miller PL. Artificial intelligence research in anesthesia and intensive care. J Clin Monit. 1988;4:274-89. https://doi. org/10.1007/BF01617327.

11. Gostt RK, Rathbone GD, Tucker AP. Real-time pulse oximetry artifact annotation on computerized anaesthetic records. J Clin Monit Comput. 2002;17:249-57. https://doi.org/10.1023/a:1020785323 889.

12. Liem VGB, Hoeks SE, van Lier F, de Graaff JC. What we can learn from Big Data about factors influencing perioperative outcome. Curr Opin Anaesthesiol. 2018;31:723-31. https://doi.org/10.1097/ ACO.0000000000000659.
13. Simpao AF, Gálvez JA. Reporting of observational research in Anesthesiology: the importance of data quality: trust but verify. Anesthesiology. 2018;128:250-1. https://doi.org/10.1097/ALN.0000000000 002007.

14. Hashimoto DA, Witkowski E, Gao L, Meireles O, Rosman G. Artificial intelligence in anesthesiology: current techniques, clinical applications, and limitations. Anesthesiology. 2020;132:379-94. https://doi.org/10.1097/ALN.0000000000002960.

15. Lonsdale H, Jalali A, Ahumada L, Matava C. Machine learning and artificial intelligence in pediatric research: current state, future prospects, and examples in perioperative and critical care. J Pediatr. 2020;221S:S3-S10. https://doi.org/10.1016/j.jpeds.2020.02.039.

16. Lee CK, Hofer I, Gabel E, Baldi P, Cannesson M. Development and validation of a deep neural network model for prediction of postoperative in-hospital mortality. Anesthesiology. 2018;129:649-62. https://doi.org/10.1097/ALN.0000000000002186.

17. Pasma W, Wesselink EM, van Buuren S, de Graaff JC, van Klei WA. Artifacts annotations in anesthesia blood pressure data by man and machine. J Clin Monit Comput XX: XXX-XX; 2020.

18. Pasma W, Peelen LM, van Buuren S, van Klei WA, de Graaff JC. Artifact processing methods influence on intraoperative hypotension quantification and outcome effect estimates. Anesthesiology. 2020;132:723-37. https://doi.org/10.1097/ALN.000000000000313 1.

19. Chen L, Dubrawski A, Wang D, et al. Using supervised machine learning to classify real alerts and artifact in online multisignal vital sign monitoring data. Crit Care Med. 2016;44:e456-e463463. https ://doi.org/10.1097/CCM.0000000000001660.

20. Du CH, Glick D, Tung A. Error-checking intraoperative arterial line blood pressures. J Clin Monit Comput. 2019;33:407-12. https://doi. org/10.1007/s10877-018-0167-7.

21. Hravnak M, Chen L, Dubrawski A, Bose E, Clermont G, Pinsky MR. Real alerts and artifact classification in archived multi-signal vital sign monitoring data: implications for mining big data. J Clin Monit Comput. 2016;30:875-88. https://doi.org/10.1007/s1087 7-015-9788-2.

22. Debayle J, Hatami N, Gavet Y. Classification of time-series images using deep convolutional neural networks. 2018; 23. $10.1117 / 12.2309486$.

23. Russell-Buckland J, Bale G, de Roever I, Tachtsidis I. ABroAD: A Machine Learning Based Approach to Detect Broadband NIRS Artefacts. Adv Exp Med Biol. 2018;1072:319-24. https://doi. org/10.1007/978-3-319-91287-5_51.

24. Thakkar P. The removal of motion artifacts from non-invasive blood pressure measurements. Electronic Theses and Dissertations, 2004 2019. 248. https://stars.library.ucf.edu/etd/248

25. Bose SN, Verigan A, Hanson J, et al. Early identification of impending cardiac arrest in neonates and infants in the cardiovascular ICU: a statistical modelling approach using physiologic monitoring data. Cardiol Young. 2019;29:1340-8. https://doi.org/10.1017/S1047 951119002002.

26. Pachauri A, Bhuyan M. Wavelet transform based arterial blood pressure waveform delineator. Int J Biol Biomed Engin. 2012;1:15-25.

27. Rush B, Celi LA, Stone DJ. Applying machine learning to continuously monitored physiological data. J Clin Monit Comput. 2019;33:887-93. https://doi.org/10.1007/s10877-018-0219-z.

Publisher's Note Springer Nature remains neutral with regard to jurisdictional claims in published maps and institutional affiliations. 